\title{
Development of Artificial Cerebrospinal Fluid: Basic Experiments, and Phase II and III Clinical Trials
}

\author{
Ryuzo Shiobara ${ }^{1 *}$, Takayuki Ohira ${ }^{1}$, Kazuhisa $\mathrm{Doi}^{2}$, Masuhiro Nishimura ${ }^{2}$ and Takeshi Kawase ${ }^{1}$
}

${ }^{1}$ Department of Neurosurgery, School of Medicine, Keio University, Tokyo, Japan

${ }^{2}$ Research and Development Center; Otsuka Pharmaceutical Factory, Inc, Tokushima, Japan

\begin{abstract}
Objectives: The first artificial cerebrospinal fluids (ACSFs), Elliot's solutions A and B, were first reported in 1949. In 1974, we developed an ACSF, ACSF trial product No. 1, and have safely used and developed this ACSF since then (No.1 to No.19). In parallel with this, after performing basic experiments and clinical trials, we obtained marketing approval for a commercially available ACSF (ARTCEREB) from the Japanese Ministry of Health, Labor, and Welfare in 2008. We now present the results of those basic experiments and clinical studies, including the phase II and III trials on ARTCEREB, with special reference to its safety and efficacy.

Methods: The composition and properties of ARTCEREB are as the same as human CSF, excluding the $\mathrm{Cl}$ concentration. Experiments with rats: the significance of bicarbonate in ACSF, and the influence and cytotoxicity of ARTCEREB, lactated Ringer's solution, and normal saline were assessed in cultured brain cells derived from rat fetuses by examining mitochondrial activity and morphological changes. In rats with brain injury, we examined the influence of these three irrigation and perfusion fluids on edema, vascular permeability, and mitochondrial activity. Clinical trials: we performed the phase II and III clinical trials on 157 patients in 17 institutions from 2002 through 2005.

Results: Experiments with rats: the morphological changes observed in the ARTCEREB-treated group were less than in the other two groups. Mitochondrial activity was higher in the ARTCEREB group than observed for the other two solutions. Mitochondrial activity in the ARTCEREB, lactated Ringer's solution, and normal saline groups was approximately $10 \%, 50 \%$, and $70 \%$, respectively, lower than observed in intact cultured brain cells. There was a significant reduction of edema and vascular permeability at the injured site in the ARTCEREB group compared to the other two groups. Mitochondrial activity was significantly higher in the ARTCEREB group than in the other two groups, indicating the lower cytotoxicity of ARTCEREB. Clinical trials: the only adverse effects associated with the use of ARTCEREB were a slightly increased temperature in two patients and decreased alkaline phosphatase activity in two patients, indicating the safety and efficacy of ARTCEREB.
\end{abstract}

Conclusions: In the present study, basic experiments on cultured brain cells derived from rat fetuses and in the injured rat brain, combined with phase II and III clinical trials demonstrated the safety and efficacy of ARTCEREB.

Keywords: Artificial cerebrospinal fluid; Irrigation fluid; Minimally invasive surgery; Neuroendoscopic surgery; Perfusion fluid

Abbreviation: ACSF: Artificial cerebrospinal fluid; CSF: Cerebrospinal Fluid; ARTCEREB: Brand Name of the Artificial Cerebrospinal Fluid; EB: Evans Blue; NIH: National Health Insurance; osm prss: Osmotic Pressure; TTC: 2,3,5-Triphenyltetrazolium Chloride; WNL: Within Normal Limits; Pharmaceutical Manufacturer: Otsuka Pharmaceutical Factory, Inc. Tokushima, Japan.

In 1949, Elliott [1] reported the first artificial cerebrospinal fluids (ACSFs), Elliott's solutions A and B. Although 60 years have passed since then, ACSFs are still not widely used because the development cost is too high and there is a low recognition of the usefulness of ACSFs by neurosurgeons. In 1974, we developed the ACSF hospital trial product No.1 in the Department of Neurosurgery, School of Medicine, Keio University, Japan. Since then, this ACSF has been continually developed up to trial product No. 19 and these products were used until 2008. During this 34-year period, these ACSFs have been safely used on 10,000 patients undergoing neurological surgery [2-6].

In parallel with this, we have developed an ACSF product, ARTCEREB, in collaboration with a pharmaceutical manufacturer, and conducted a variety of studies on ARTCEREB including basic experiments [7,8], studies on its use as an irrigation fluid for neurosurgery $[9,10]$ and in phase II [11] and III [12] clinical trials. In 2008, the Japanese Ministry of Heath, Labor, and Welfare approved the marketing of ARTCEREB (NHI drug price listing). It is expected that ARTCEREB will be extensively used worldwide in the fields of neurosurgery and neurosciences.

\section{Methods}

\section{Artcereb}

The safety and efficacy of ARTCEREB were comparatively studied with other irrigation and perfusion fluids, e.g., lactated Ringer's solution and normal saline, in basic experiments, and in phase II and III clinical trials. The composition and properties of ARTCEREB are shown in Table 1, and the composition of human CSF reported by Davson [13] and Milhorat [14] is shown in Table $1 . \mathrm{Na}^{+} 145.4(145.5) \mathrm{mEq} / \mathrm{L}, \mathrm{K}^{+}$ $2.8(2.8) \mathrm{mEq} / \mathrm{L}, \mathrm{Mg}^{2+} 2.2(2.2) \mathrm{mEq} / \mathrm{L}, \mathrm{Ca}^{2+} 2.3(2.3) \mathrm{mEq} / \mathrm{L}, \mathrm{Cl}^{-} 128.5$ (111.9) mEq/L, $\mathrm{HCO}_{3}-23.1(23.1) \mathrm{mEq} / \mathrm{L}, \mathrm{P} 1.1$ (1.1) mmol/L, glucose

*Corresponding author: Ryuzo Shiobara, Department of Neurosurgery, Keio University, School of Medicine, 35 Shinanomachi, Shinjuku-Ku, Tokyo 160-8582 Japan, Tel: +81(3) 3353-1211; E-mail: shiobara@1961.jukuin.keio.ac.jp

Received October 08, 2013; Accepted November 26, 2013; Published December 04, 2013

Citation: Shiobara R, Ohira T, Doi K, Nishimura M, Kawase T (2013) Development of Artificial Cerebrospinal Fluid: Basic Experiments, and Phase II and III Clinical Trials. J Neurol Neurophysiol 4: 173. doi:10.4172/2155-9562.1000173

Copyright: @ 2013 Shiobara R, et al. This is an open-access article distributed under the terms of the Creative Commons Attribution License, which permits unrestricted use, distribution, and reproduction in any medium, provided the original author and source are credited. 


\begin{tabular}{|c|c|c|c|c|c|}
\hline Component & Unit & $\begin{array}{l}\text { Human } \\
\text { normal } \\
\text { CSF }\end{array}$ & ARTCEREB & Normal saline & $\begin{array}{l}\text { Lactated } \\
\text { Ringer's } \\
\text { solution }\end{array}$ \\
\hline $\mathrm{Na}^{+}$ & $\mathrm{mEq} / \mathrm{L}$ & 145.5 & 145.4 & 154 & 130.0 \\
\hline $\mathrm{K}^{+}$ & $\mathrm{mEq} / \mathrm{L}$ & 2.8 & 2.8 & --- & 4.0 \\
\hline $\mathrm{Mg}^{+}$ & $\mathrm{mEq} / \mathrm{L}$ & 2.2 & 2.2 & ---- & --- \\
\hline $\mathrm{Ca}_{2}^{+}$ & $\mathrm{mEq} / \mathrm{L}$ & 2.3 & 2.3 & ---- & ---- \\
\hline $\mathrm{Cl}^{-}$ & $\mathrm{mEq} / \mathrm{L}$ & 111.9 & 128.5 & 154 & 109.0 \\
\hline $\mathrm{HCO}_{3}^{-}$ & $\mathrm{mEq} / \mathrm{L}$ & 23.1 & 23.1 & --- & $28.0^{* * *}$ \\
\hline $\mathrm{P}$ & $\mathrm{mmol} / \mathrm{L}$ & 1.1 & 1.1 & ---- & ---- \\
\hline Glucose & $\mathrm{g} / \mathrm{L}$ & $0.61^{*}$ & 0.61 & --- & --- \\
\hline $\begin{array}{l}\text { Osmotic ratio* } \\
\text { approx.0.9 }\end{array}$ & & $\begin{array}{l}\mathrm{mosm} / \mathrm{kg} \\
\mathrm{H}_{2} \mathrm{O}\end{array}$ & approx. 1 & approx. 1 & 1 \\
\hline $\mathrm{pH}$ & & 7.307 & $\fallingdotseq 7.3$ & $4.5 \sim 8.0$ & $6.0 \sim 8.5$ \\
\hline
\end{tabular}

"Davson, "Milhorat, "*tactate. [Modified and translated with permission from reference [11]]

Table 1: Composition of test solution and human normal CSF

$0.61(0.61) \mathrm{g} / \mathrm{L}$, osmotic ratio $\fallingdotseq 1(289 \mathrm{mOsm} / \mathrm{kg}), \mathrm{pH} \fallingdotseq 7.3(7.307)$ (Table 1). Figure 1 shows the relative concentration of each component in ARTCEREB, lactated Ringer's solution, and normal saline to the corresponding component in human CSF (defined as 100).

ARTCEREB is contained in a double compartment bag to suppress the interaction of the constituents; the bag is further packed within a $\mathrm{CO}_{2}$ barrier free outer bag to maintain the $\mathrm{pH}$ stability. To use ARTCEREB, the inner bag (the double compartment bag) is removed from the outer bag and the septum is opened by manual compression to mix the two fluids. Although ARTCEREB was infused or dripped from an infusion set into the operative field, its $\mathrm{pH}$ was kept within the allowable range (below $\mathrm{pH} 7.8$ ) when stored in an open system, such as a bowl, for 6 hours at room temperature (Figure 2).

\section{Animal preparation}

This study was approved by the Otsuka Pharmaceutical Factory Committee on the Care and Use of Laboratory Animals and was conducted in accordance with in-house guidelines that follow the Guide for the Care and Use of Laboratory Animals (US National Research Council). Two sets of basic experiments were conducted in rats.

\section{Evaluation of the significance of bicarbonate in irrigation and perfusion fluids on the mitochondrial activity of cultured brain cells derived from rat fetuses}

A primary culture system of rat fetus brain cells [8] was used. Brains were isolated from rat fetuses on the $15-16^{\text {th }}$ day of pregnancy under ether anesthesia. A suspension of viable cells, approximately 1.0 $\times 10^{5}$ cells $/ \mathrm{mL}$, was prepared from the brain tissue. Cell preparations in which the viability was higher than $90 \%$ were used in the experiments. The cell suspension was dispensed into a 6-well plate in aliquots of 2 $\mathrm{mL} /$ well or into a 24 -well plate in aliquots of $0.4 \mathrm{~mL} /$ well. The cells were cultured in a $5 \% \mathrm{CO}_{2}$ incubator. The culture medium was exchanged on the $3^{\text {rd }}$ and $5^{\text {th }}$ days, and cells were used on the $6^{\text {th }}$ to $7^{\text {th }}$ day after plating.

Rhodamine 123 which is easily incorporated into cells is specifically distributed to the mitochondria, and fluorescently stains these organelles [15]. Cultured brain cells were exposed to the test solutions for 3 hours. The mitochondria were fluorescently stained with rhodamine 123 and microscopically observed 24 hours after exposure
(Figure 3). In addition, the amount of rhodamine 123 incorporated into the cultured cells was measured and we calculated the relative amount of rhodamine 123 in cells exposed to the test solutions with a comparison to the intact cultured cells (Figure 4).

\section{Cellular damage and edema in injured rat brains}

We used 88 Sprague-Dawley rats, weighing 290-300 g (Charles River Japan, Inc., Yokohama, Japan) to study brain edema, cerebrovascular permeability and mitochondrial activity of damaged brain tissue. Animals were randomly allocated to the normal saline group, the lactated Ringer's solution group, or the ARTCEREB group. Rats were anesthetized by the intraperitoneal injection of urethane, and were positioned in a stereotactic frame (SR-6N; Narishige Scientific Instrument Laboratory, Tokyo, Japan). A burr hole opening (4 mm diameter), the removal of the dura mater and arachnoid membrane, and a $1.5 \mathrm{~mm}$ depth and $3.5 \mathrm{~mm}$ length wound were made. The wounds were separately irrigated with $150 \mathrm{~mL} / \mathrm{hr}$ of the 3 test fluids for 4 hours. The irrigation solutions were administered in a blinded manner. The rats were kept warm with a feedback-controlled lamp and a warming pad to maintain a rectal temperature of approximately $37^{\circ} \mathrm{C}$ until the end of the irrigation period (Figure 5).

Part 1: determination of the specific gravity of brain tissue: After the irrigation period, the rats were killed by exsanguination from the abdominal aorta, and the brains were quickly removed. To prevent evaporative water loss, we temporarily stored each brain in cooled

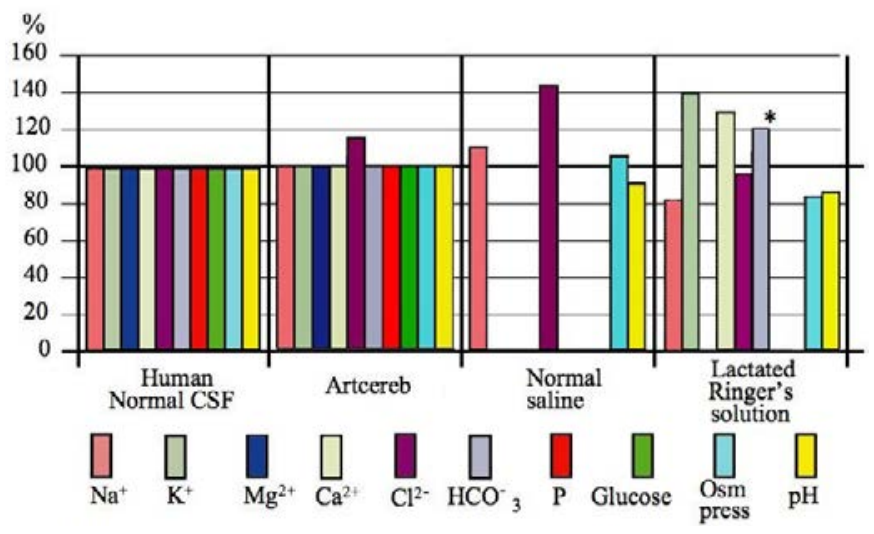

Figure 1: Ratio of composition of solutions to human normal CSF. *Lactated Ringer's solution contains lactate- $(28 \mathrm{mEq} / \mathrm{L})$ instead of $\mathrm{HCO}_{3}^{-}(23.1 \mathrm{mEq} / \mathrm{L})$ in ARTCEREB.

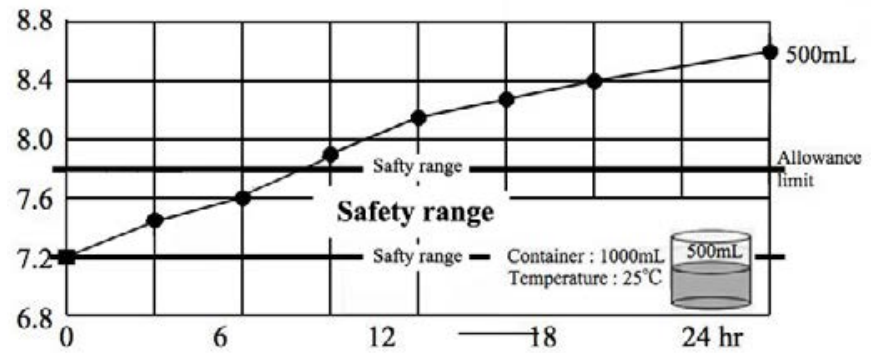

Figure 2: Time course change in $\mathrm{pH}$ of ARTCEREB after transferring into open container. 
Citation: Shiobara R, Ohira T, Doi K, Nishimura M, Kawase T (2013) Development of Artificial Cerebrospinal Fluid: Basic Experiments, and Phase II and III Clinical Trials. J Neurol Neurophysiol 4: 173. doi:10.4172/2155-9562.1000173

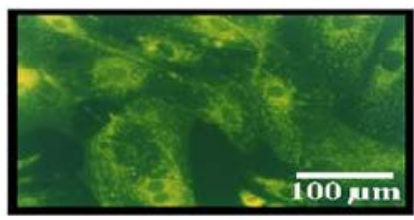

Control

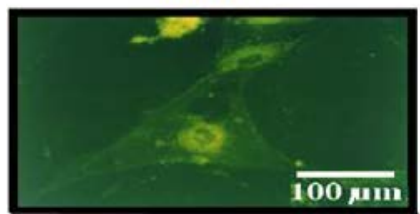

Lactated Ringer's solution

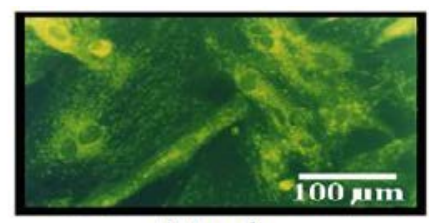

Artcereb

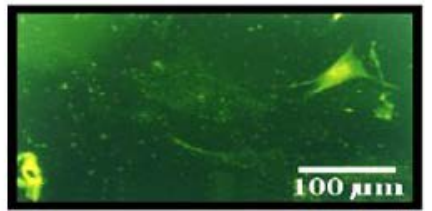

Normal saline
Figure 3: Effects of various test solutions on the cytotoxity of cultured rat brain cells. Evaluation of mitochondrial activity. [Reprinted with permission from reference [8]].

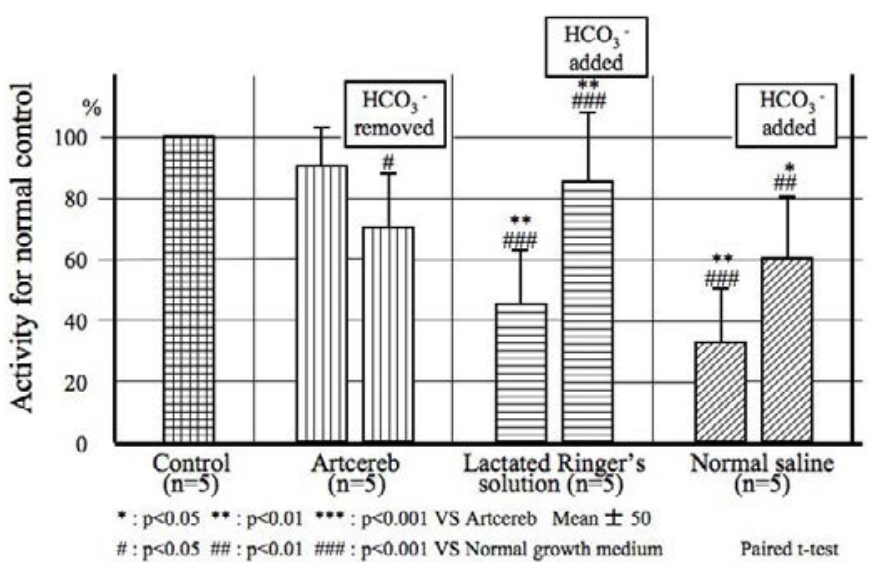

Figure 4: Effects of bicarbonate on mitochondrial activity rat fetal brain cells. [Modified with permission from reference [8]].

kerosene (Wako Pure Chemical Industries, Ltd, Osaka, Japan) until the tissues were sampled. Brain tissue samples, measuring approximately $0.5 \mathrm{~mm}^{3}$ of size, were obtained from the area facing the intersection of the wounds (injured site) and from the area symmetrically opposite to the injured site in the uninjured contralateral hemisphere (uninjured site).

The samples were placed in a gravimetric column of bromobenzene (Wako Pure Chemical Industries, Ltd) and kerosene (Wako Pure Chemical Industries, Ltd) of a known density, following the method of Marmarou et al. [16]. The column was calibrated with potassium sulfate solutions (Wako Pure Chemical Industries, LTD), with specific gravities of 1.020,1.029, 1.038, 1.047, and 1.056, before use. The measured data were placed over the calibration curve, and the corresponding specific gravity values were read and recorded accordingly.

Part 2: quantitative evaluation of Evans Blue extravasation: Two percent Evans Blue (EB) dye in saline, was given intravenouslyat a dose of $5 \mathrm{~mL} / \mathrm{kg}$ as a cerebrovascular permeability tracer 3 hours after the injury in the ARTCEREB, lactated Ringer's solution, and normal saline groups. The irrigation was stopped 4 hours after the injury. Animals were perfused with approximately $250 \mathrm{~mL}$ of normal saline through the left ventricle at $100 \mathrm{~cm}$ of water pressure to remove the intravascularly localized dye. After decapitation, the brain was removed and examined for evidence of EB staining, and then separated into cortices and basal ganglia. Cortices were flattened on a plate, and, using a 4-mm-diameter cork borer, brain tissue samples were obtained from the injured and uninjured sites. The extraction and determination of the EB dye were performed according to the modified method reported by Uyama et al. [17] Brain samples were weighed and homogenized in a 10-fold volume of phosphate buffer and mixed with a 10 -fold volume of $50 \%$ trichloroacetic acid to precipitate the proteins. The supernatant was obtained by centrifugationand diluted 4-fold with ethanol. Fluorescence was determined (excitation at $620 \mathrm{~nm}$ and emission at $680 \mathrm{~nm}$ ) with a spectrofluorometer (FP-750, JASCO Co, Tokyo, Japan). The calculations were based on external standards of the solvent (25$500 \mathrm{ng} / \mathrm{mL}$ ). The tissue content of EB was expressed as micrograms per gram of brain tissue. Rats in the control group was administered with EB 3 hours after body temperature maintenance was initiated, and they underwent the same operation as did the other groups.

Part 3: TTC incubation and measurement of tissue formazan levels: 2,3,5-Triphenyltetrazolium chloride (TTC) is reduced in surviving tissue by mitochondrial succinate dehydrogenase to a red formazan product. TTC staining of brain tissue followed by solvent extraction and spectrophotometric measurement of formazan can, therefore, provide an objective index of experimental brain injury. We performed TTC staining and measurement of tissue formazan

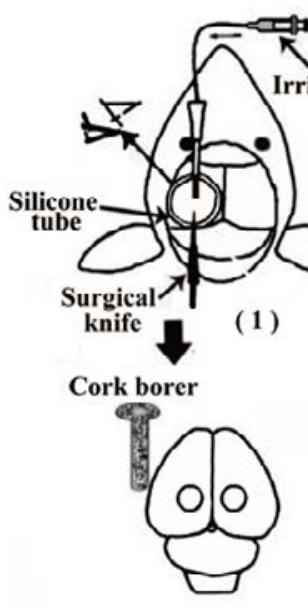

(5)

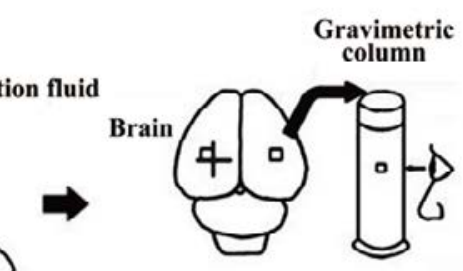

(2)

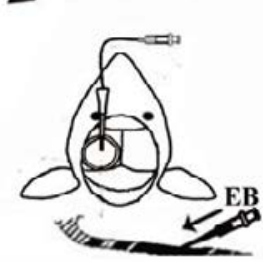

(3)

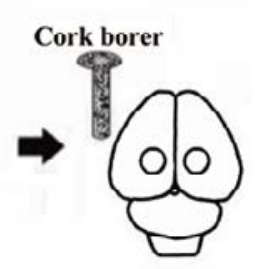

(4)
Figure 5: Schematic diagram of the experimental protocol. An burr hole was made in the left side of the skull through the silicone tube. Via the burr hole, irrigation with each solution was started, and the dura mater, arachnoid membrane, and cerebral cortex were incised crosswise in the area of the opening with a microsurgical knife. The dura mater and arachnoid mater were removed from the injured area (1). Part 1: Four hours after injury, brain tissue samples were obtained from the area facing the intersection of the wound (injured site) and from the area asymmetrically opposite to the injured site in the uninjured contralateral hemisphere (uninjured site). Samples were then placed in a gravimetric column (2). Part 2: Two percent of EB in saline was given intravenously 3 hours after injury (3). Four hours after injury, irrigation was stopped. To remove the intravascularly localized dye, we perfused with normal saline. Using 4-mm-diameter cork borer, brain tissue samples were obtained from the area of the wounds (injured site) and from the area symmetrically opposite to the injured site in the uninjured contralateral hemisphere (uninjured site). Extraction and determination of EB dye were then performed (4). Part 3 : Using a 4-mm-diameter cork borer, brain tissue samples were obtained from the area of the wounds (injured site) and from the area symmetrically opposite to the injured site in the uninjured contralateral hemisphere (uninjured site). Then, TTC incubation and measurement of tissue formazan were performed (5). [Modified with permission from reference [7]]. 
levels in brain tissue according to the modified method reported by Preston and Webster [18]. The irrigation was stopped 4 hours after the injury. The animals were decapitated, and their heads were chilled on ice for approximately 30 seconds. The brains were removed and separated into cortices and basal ganglia. Cortices were flattened on an ice-cooled plate, and, using a 4 -mm-diameter cork borer, brain tissue samples were obtained from the injured and uninjured sites. Each tissue sample was cut in half, and weighed in pre-tared liquid scintillation vials. A bathing solution, containing $140 \mathrm{mmol} / \mathrm{L}$ sodium chloride, $5 \mathrm{mmol} / \mathrm{L}$ potassium chloride, $1 \mathrm{mmol} / \mathrm{L}$ calcium chloride, $10 \mathrm{mmol} / \mathrm{L}$ HEPES, and $3 \mathrm{mmol} / \mathrm{L}$ glucose, was used to dissolve the TTC ( $2 \%$ solution), and $5 \mathrm{~mL}$ was added to each tissue vial. The vials were incubated for 90 minutes at $37^{\circ} \mathrm{C}$. The TTC was then removed, and the tissue was rinsed twice with normal saline. Approximately $5 \mathrm{~g}$ of a 50:50 mixture of ethanol/dimethyl sulfoxide was added to solubilize the formazan. The vials were tightly capped and placed in the dark for 24 hours. For analysis, the red solvent extract was placed in a cuvette and the absorbance of this cuvette was measured at $485 \mathrm{~nm}$ in a spectrophotometer (V-550, JASCO Co).

\section{Statistical analysis}

The experimental data are expressed as the mean \pm SD. Statistical analysis was performed using Dunnett's test for multiple comparisons to evaluate the intergroup differences. Differences were considered to be statistically significant at $P<0.05$.

\section{Clinical trials}

The clinical trials were conducted as non-randomized and nonblind open studies according to the ethical principles of the Declaration of Helsinki [19] (Helsinki, Finland, June 1964), the standards provided by Article 14 Paragraph 3 and Article 80 Paragraph 2 of the Japanese Pharmaceutical Affairs Law (August 10, 1960: Law No. 145) [20], and the Ordinance of the Ministry of Health and Welfare for the implementation standards for the clinical tests of drugs (March 27, 1997: Ordinance of the Ministry of Health and Welfare, Japan, No. 28) [21].
Phase II clinical trial [11]: Although lactated Ringer's solution and normal saline have been used for intracranial irrigation, intraventricular perfusion, and infusion during neurological surgery, such usage has not been officially approved in Japan. Since this clinical trial was the first trial in which ARTCEREB was applied to humans, the trial was separated into two steps and performed as follows: step 1 was sequentially carried out in patients undergoing mild, moderate, and severe surgical invasion in a stepwise manner to confirm the safety of ARTCEREB; and step 2 was carried out with no thought to the severity of the surgical infusion.

Patients who met any of the criteria listed in Table 2 were excluded from the study. We studied 44 patients aged between 25 and 75 years in 3 institutions. Forty patients underwent neurological surgery with a burr hole opening and craniotomy, and four patients underwent neuroendoscopic surgery. Safety was evaluated based on the primary and secondary evaluation endpoints shown in Table 3. Collected data were analyzed from the medical viewpoint in comparison with clinical survey samples. To determine the clinical efficacy (performance) of ARTCEREB in practical use, we evaluated: (1) its capacity to irrigate an operative field, (2) its capacity to exclude air from the operative field, and (3) its influence on a surgical coagulation device (electric coagulator) in patients undergoing neurological surgery with a burr hole opening and craniotomy. We also assessed: (1) its capacity to secure the cleanliness of the operative field, (2) the adhesive feeling of the perfusion fluid, and (3) its influence on a surgical coagulation device in patients undergoing neuroendoscopic surgery.

Phase III clinical trial [12]: Patients who met any of the exclusion criteria (Table 2) were excluded. A safety evaluation was performed in 113 patients in 17 institutions for the endpoints shown in Table 3. Subjects included 98 patients who underwent neurological surgery with a burr hole opening and craniotomy, and 15 patients who underwent neuroendoscopic surgery. In addition, we surveyed the clinical efficacy (performance) of ARTCEREB in practical use among the surgeons for the same items used in the phase II clinical trial.

Adverse events and judgment of adverse effects: any unfavorable

1) Patients having severe hepatic disorder (patients having any of following criteria)

Total bilirubin level is higher than $2.3 \mathrm{mg} / \mathrm{dL}$.

AST level is higher than 3.0x the upper limit of normal range of the institution.

ALT level is higher than 3.0x the upper limit of normal range in the institution.

2) Patient having severe renal disorder (patients having any of following criteria).

BUN level is higher than $40 \mathrm{mg} / \mathrm{dL}$.

Creatinine level is higher than $40 \mathrm{mg} / \mathrm{dL}$.

3) Patients having severe heart failure (Patients with NYHA classification IV ${ }^{3}$ )

4) Pregnant patients or possibly pregnant patients (Patients who are diagnosed as positive in pregnancy test [except for pregnant or post-menopausal patients])

5) Patients who are infected with hepatitis $B$, hepatitis $C$ or syphilis

6) Patients who underwent blood collection (blood donation) of more than $200 \mathrm{ml}$ with a month, or of more than $400 \mathrm{ml}$ within 3 months counted from the scheduled operation date (except for collection for autologous blood transfusion)

7) Patients who participated in other clinical trials within 6 months counted from the scheduled operating date

8) Patient who underwent craniotomy within a month counted from the scheduled operation date

9) Patients who are judged not to be eligible by principal and sub investigators because of expected difficulty in compliance of protocol etc.

Table 2: Subjects of the clinical study (Exclusion criteria). [Modified and translated with permission from reference [11]]

\begin{tabular}{|c|c|}
\hline Primary endpoint & Clinical symptoms endpoint other than primary endpoints \\
\hline Axillary temperature & Clinical symptoms \\
\hline Headache & Neurological findings \\
\hline Nausea & Vital signs \\
\hline Vomiting & Hematological examination \\
\hline Consciousness $\left(\mathrm{JCS}^{*}\right)$ & Blood biochemical examination \\
\hline Blood Cl concentration & \\
\hline
\end{tabular}

Table 3: Endpoints for safety evaluation. JCS ${ }^{*}=J a p a n$ Coma Scale [33,3]: [Modified and translated with permission from reference [11]]. 
Citation: Shiobara R, Ohira T, Doi K, Nishimura M, Kawase T (2013) Development of Artificial Cerebrospinal Fluid: Basic Experiments, and Phase II and III Clinical Trials. J Neurol Neurophysiol 4: 173. doi:10.4172/2155-9562.1000173

or unintended signs, symptoms, or diseases (including abnormal laboratory values) associated with the use of ARTCEREB were considered as adverse events. An adverse event whose causal relationship with the use of ARTCEREB could not be refuted was judged to be an adverse effect.

Data collection and statistical analysis: the efficacy of ARTCEREB was evaluated by the frequency distribution categorized into four levels. Data were collected on the frequency distribution, adverse events and their incidence, the incidence of intracranial complications among adverse events, and the degree and frequency of systemic complications. Descriptive statistics were calculated for indiscrete values.

\section{Results}

Evaluation of the influence of the irrigation and perfusion fluids on the mitochondrial activity of cultured brain cells derived from rat fetuses

Microscopic observation of cultured brain cells derived from rat fetuses, which were fluorescently stained with rhodamine 123 , revealed that there were no differences in the amount of incorporated rhodamine123 in the cells and mitochondria of the ARTCEREB group and intact cultured cells. On the other hand, the lactated Ringer's solution and normal saline groups showed decreased rhodamine 123 incorporation into cells and mitochondria, a decreased number of cells, and more severe morphological changes in viable cells in comparison with the ARTCEREB group (Figure 3). Cultured cells treated with ARTCEREB, which contained bicarbonate and a $\mathrm{pH}$ adjusted to 7.4, showed higher rhodamine 123 uptake than the lactated Ringer's solution and normal saline groups; the mitochondrial activity in the lactated Ringer's solution and normal saline groups was $45 \%$ and $32 \%$, respectively, lower than observed in the intact cultured cells (Figure 4). In addition, although the mitochondrial activity of cells exposed to ARTCEREB was similar to that of intact cultured cells 24 hours after exposure, the mitochondrial activity of cells exposed to bicarbonatefree ARTCEREB was significantly lower than that observed in the intact cultured cells.

\section{Specific gravity of injured brain tissue}

The specific gravities of the injured sites were significantly lower in the normal saline $(1.025 \pm 0.001)$ and lactated Ringer's solution (1.031 $\pm 0.002)$ groups than in the ARTCEREB group $(1.035 \pm 0.003 ; P<0.001$ and $P<0.01$, respectively). The specific gravities of the uninjured sites in the ARTCEREB (1.049 \pm 0.001$)$, lactated Ringer's solution (1.048 \pm $0.001)$, and normal saline $(1.049 \pm 0.001)$ groups were similar, but they were higher than that observed in the injured sites (Figure 6).

\section{Evans blue (EB) extravasation of injured brain tissue}

Examination of the brain 4 hours after injury revealed a bluestained area at the site of injury in the ARTCEREB, lactated Ringer's solution, and normal saline groups; no staining was noted in the control group (Figure 7).

The concentration of EB in the injured sites of the ARTCEREB $(10.5 \pm 1.3)$, lactated Ringer's solution $(15.3 \pm 3.9)$, and normal saline $(13.8 \pm 2.9)$ groups, was significantly higher than in the control group ( $4.9 \pm 0.4 ; P<0.001$ for each). The concentration of EB was significantly higher in the injured sites of the lactated Ringer's solution group $(P<0.01)$ and relatively higher in the normal saline group as compared with the ARTCEREB group. The concentration of EB in the uninjured sites in the control $(4.7 \pm 0.4)$, ARTCEREB $(4.7 \pm 0.4)$, lactated Ringer's solution $(5.0 \pm 1.0)$, and normal saline $(4.7 \pm 0.4)$ groups was not significantly different (Figure 8).

\section{TTC staining of injured brain tissue}

TTC staining of the injured sites did not differ significantly between the control $(0.242 \pm 0.017)$ and ARTCEREB $(0.220 \pm 0.023)$ groups. TTC staining of the injured sites in the lactated Ringer's solution $(0.189$ $\pm 0.023)$ and normal saline $(0.168 \pm 0.030)$ groups was significantly lower than in the control group $(P<0.001$ for each). TTC staining of the injured sites in the lactated Ringer's solution and normal saline groups was significantly lower when compared to the ARTCEREB group $(P<0.05$ and $P<0.01$, respectively). TTC staining of the uninjured sites in the control $(0.244 \pm 0.014)$, ARTCEREB $(0.254 \pm 0.020)$, lactated Ringer's solution $(0.237 \pm 0.016)$, and normal saline $(0.232 \pm 0.018)$ groups was not statistically different (Figure 9).

\section{Clinical trials: phase II and phase III}

The backgrounds of the study subjects of phase II and phase

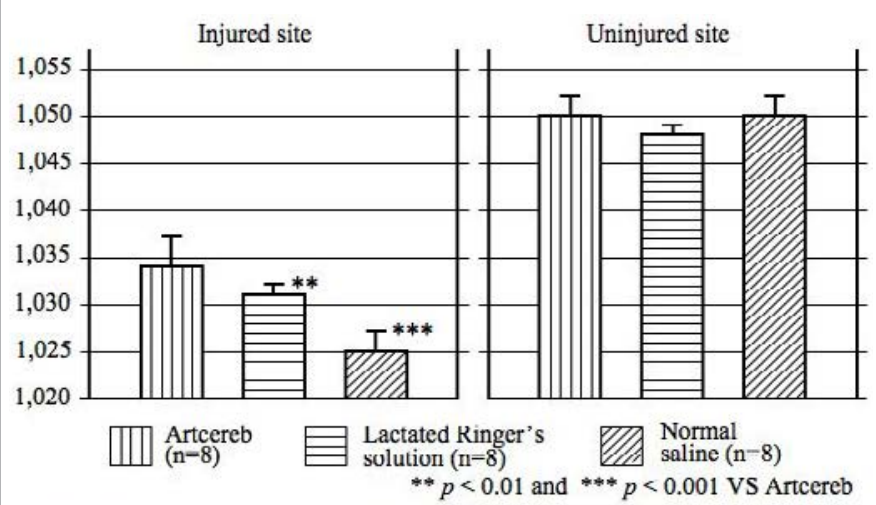

Figure 6: Specific gravity of brain tissue 4 hours after injury. Data are expressed as mean \pm SD. Statistical analysis was performed using Dunnett's test for multiple comparisons to evaluate intergroup differences in the injured site. [Modified with permission from reference [7]].

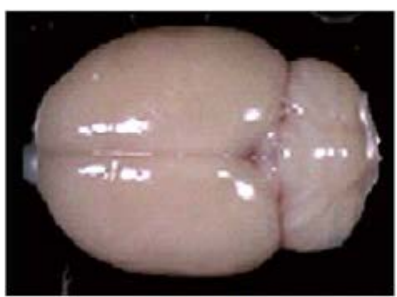

Control

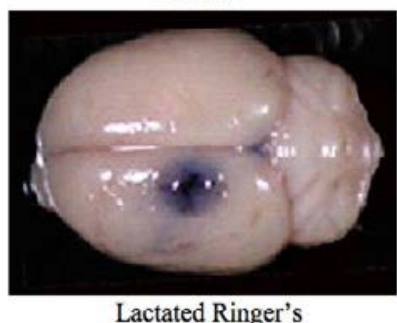
solution

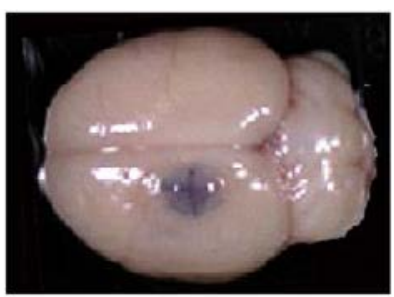

Artcereb

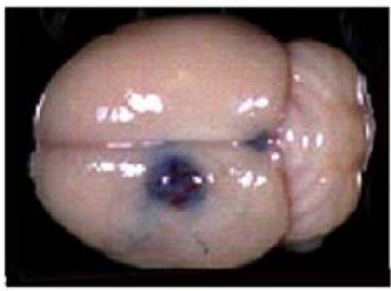

Normal saline
Figure 7: Gross appearance of the brain 4 hours after injury. In the control group, no blue-stained area was noted. In the Artcereb group, lactated Ringer's solution group, and normal saline group, a blue-stained area was seen at the site of injury. [Modified with permission from reference [7]]. 


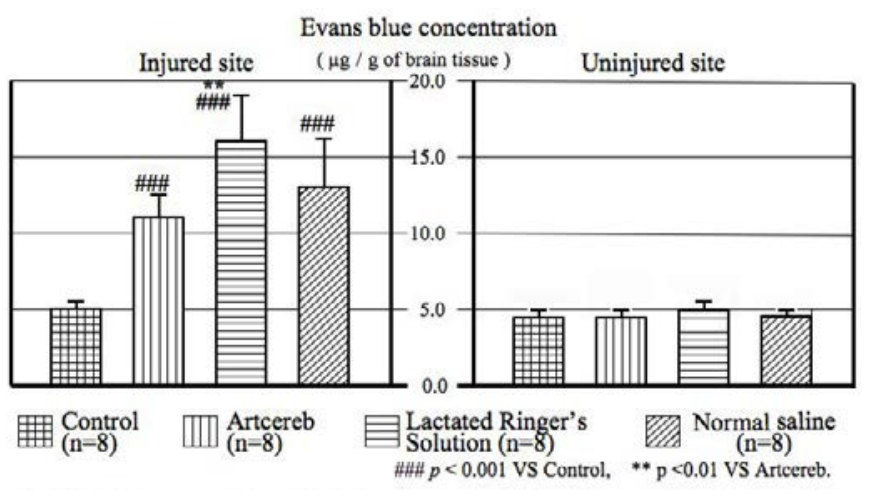

Figure 8: Evans blue concentration of brain tissue 4 hours after injury. Data are expressed as mean \pm SD. Statistical analysis was performed using Dunnett's test for multiple comparisons to evaluate intergroup differences. [Modified with permission from reference [7]]

2,3,5-triphenyltetrazolium chloride (TTC) staining ( absorbance per milligram of protein of brain tissue )

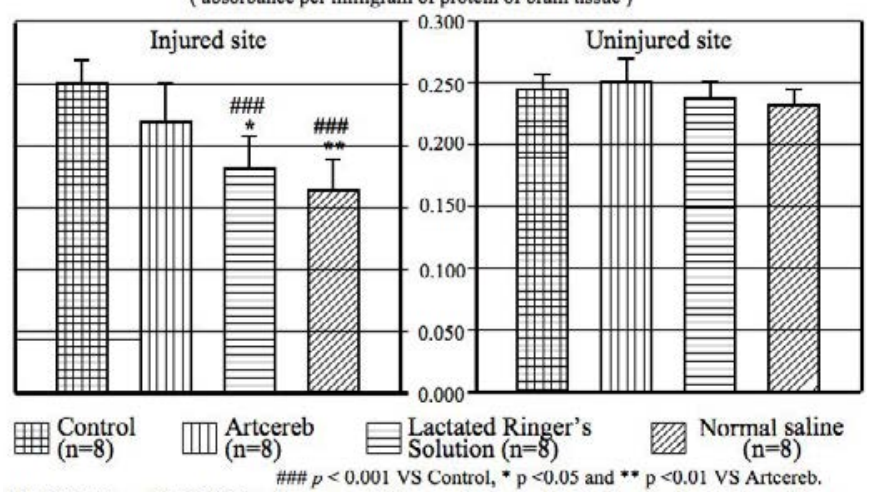

Figure 9: Staining with TTC (absorbance per milligram of protein of brain tissue) of brain tissue 4 hours after injury. Data are expressed as mean \pm SD. Statistical analysis was performed using Dunnett's test for multiple comparisons to evaluate intergroup differences. [Modified with permission from reference [7]] .

III clinical trials are shown in Table 4. The volume of ARTCEREB administered in the phase II clinical trial [11] was 100-4130 mL (mean \pm SD: $1237.1 \pm 922.5 \mathrm{~mL}$ ) and 1850-4000 mL (mean \pm SD: $2527.5 \pm$ $1014.7 \mathrm{~mL}$ ) for patients undergoing neurosurgery with a burr hole opening and craniotomy, and those undergoing neuroendoscopic surgery, respectively. In the phase III clinical trial [12], 50-8750 mL (mean \pm SD: $1283.6 \pm 1164.5 \mathrm{~mL}$ ) and $350-2980 \mathrm{~mL}$ (mean \pm SD: $1376.0 \pm 813.2 \mathrm{~mL}$ ) of ARTCEREB was used for patients undergoing neurosurgery with a burr hole opening and craniotomy, and those undergoing neuroendoscopic surgery, respectively (Table 4).

Evaluation of safety: a total of 2,745 mild, moderate, and severe adverse events were reported in patients underwent neurological surgery with a burr hole opening and craniotomy and those underwent neuroendoscopic surgery. We observed decreased alkaline phosphatase activity in two patients underwent neurological surgery with a burr hole opening and craniotomy, and a slightly increased temperature in two patients $\left(37.4^{\circ} \mathrm{C}\right.$ and $38.1^{\circ} \mathrm{C}$, respectively) underwent neuroendoscopic surgery. The decrease in alkaline phosphatase activity was judged to be an adverse effect because it was an unusual postoperative event, although it is not considered that the changes were medically significant. The slightly increased temperature was judged to be an adverse effect by the two principle physicians because the possibility of chemical (aseptic) meningitis caused by ARTCEREB could not be excluded. The severity of these adverse effects was judged to be mild, and all four patients subsequently recovered.

Evaluation of efficacy (performance): the efficacy of each of the assessed items (described in the Methods section) and the overall efficacy were judged to be good or excellent in all patients.

\section{Discussion}

\section{Composition of the irrigation and perfusion fluids used in neurological surgery}

The use of ACSFs is not yet widespread, despite a considerable number of neurosurgeons and neuroscientists who consider that ACSFs are useful. There are a number of reasons for this observation. First, hospitals that use ACSFs prepare their own as an in-hospital preparation. However, this is expensive and the hospitals have to bear this cost because the use of transfusion fluids has not been approved for intracranial irrigation and perfusion by the Ministry of Health, Labor, and Welfare. Second, pharmaceutical manufacturers have not developed ACSFs as they require a large financial investment and a long research and development period (the development requires approximately 10 years); ACSFs are also not yet profitable as the market size is small. It is considered that ARTCEREB, with a similar composition to human CSF, has become widely used because it obtained marketing approval by the Japanese Ministry of Health, Labor, and Welfare as an irrigation and perfusion fluid.

The composition and properties of ARTCEREB, which we have developed since 1974, are shown in Table 1 and Figure 1. The components and their concentrations of ARTCEREB are the same as those in human CSF except for $\mathrm{Cl}^{-}$; the concentration of $\mathrm{Cl}^{-}$in ARTCEREB is $16.6 \mathrm{mEq} / \mathrm{L}$, which is higher than that in human CSF. Figure 1 shows the relative concentration of each component in ARTCEREB, lactated Ringer's solution, and normal saline when the concentration of corresponding component in human CSF is defined as $100 \%$. The composition and properties of lactated Ringer's solution and normal saline are entirely different from those of human CSF. In addition, lactated Ringer's solution contains lactate instead of the $\mathrm{HCO}_{3}$. It has been considered that both lactated Ringer's solution and normal saline are utterly non-physiological and useless, but they have been used as irrigation and perfusion fluids throughout the history of neurological surgery.

Oka et al. [22] reported the occurrence of adverse effects associated with the use of normal saline as an irrigation and perfusion fluid, and suggested that normal saline was not appropriate for this purpose. Kodama et al. [23] did not use normal saline for ventriculo-cisternal perfusion to prevent vasospasm after subarachnoid hemorrhage. Ohira

\begin{tabular}{|c|c|c|c|c|}
\hline \multicolumn{3}{|c|}{$\begin{array}{c}\text { Patients undergoing open Burr hole and } \\
\text { craniotomy }\end{array}$} & $\begin{array}{r}\text { Patients undergoing neuroendoscopic } \\
\text { surgery }\end{array}$ \\
\hline Phase II (40pts) & $\begin{array}{c}\text { Phase III } \\
\text { (98pts) }\end{array}$ & Phase II (4pts) & Phase III (15pts) \\
\hline Mean & 1237.1 & 1283.6 & 2527.5 & 1376.0 \\
\hline SD & 922.5 & 1164.5 & 1014.7 & 813.2 \\
\hline Maximum & 4130.0 & 8750.0 & 4000.0 & 2980.0 \\
\hline Median & 1145.0 & 1000.0 & 2130.0 & 1180.0 \\
\hline Minimum & 100.0 & 50.0 & 1850.0 & 350.0 \\
\hline
\end{tabular}

pts=patients [Modified and translated with permission from reference [11]].

Table 4: Volume of ARTCEREB administered in phase I and phase III clinical trials $(\mathrm{mL})$. 
et al. [24-26] cautioned on the complications and countermeasures of neuroendoscopic surgery that normal saline should never have been used for neuroendoscopic surgery, and they used the ACSF which was prepared by our hospital for their rigid-rod neuroendoscopic and navigation endo-microscopic surgeries. Enomoto et al. [27] reported that exposure of cultured astrocytes derived from a human fetus to normal saline caused cell damage, but exposure to an ACSF only caused slight morphological changes. Uchida et al. [28] also reported that normal saline had detrimental effects, such as cell damage, on neuronal cells and mentioned that it was possibly harmful. Before the launch of ARTCEREB, some hospitals in Japan, including ours, no longer used normal saline for neurological surgery with craniotomy, and for ventriculo-cisternal perfusion and ventricular perfusion during neuroendoscopic surgery; these institutes used solutions with a similar composition to human CSF or an ACSF prepared in-hospital [20,2830]. Our basic experiments demonstrated that ARTCEREB, which is more physiological as an irrigation and perfusion fluid, caused less damage to neuronal cells than lactated Ringer's solution or normal saline $[7,8]$; our clinical studies demonstrated a favorable safety and efficacy profile of ARTCEREB [11,12]. Therefore, it is considered that ARTCEREB should be more widely used in the field of neurosurgery.

\section{Significance of bicarbonate in ACSF}

Elliott [1] and Lewis [31,32] stressed the significance of bicarbonate in ACSF as early as 1945 and 1950, respectively. We demonstrated that the effects of the irrigation and perfusion fluids on the mitochondrial activity of neuronal cells were greatly influenced by the presence or absence of bicarbonate. In cultured brain cells exposed to irrigation and perfusion fluids, ARTCEREB had significantly less unfavorable effects on mitochondrial activity and morphology than the other solutions (Figures 3 and 4). As pointed out by Lewis, bicarbonate is important because it stabilizes the $\mathrm{pH}$ of a solution. On the other hand, special care is needed for the preparation and use of ACSF because bicarbonate is labile. The bicarbonate ion, $\mathrm{HCO}_{3}^{-}$, decomposes to $\mathrm{H}_{2} \mathrm{O}$ and $\mathrm{CO}_{2}$ partiallywhen it comes into the water solution; the $\mathrm{CO}_{2}$ diffuses into the air and the $\mathrm{pH}$ of the solution rises. In addition, rises in fluid temperature during steam sterilization under pressure promotes the generation of $\mathrm{CO}_{3}{ }^{2-}$, which binds with $\mathrm{Ca}^{2+}$ and $\mathrm{Mg}^{2+}$ and further promotes binding of these ions with phosphate ions. These reactions generate insoluble salts, which cause a white clouding with a rise the $\mathrm{pH}$ of ACSF.

Therefore, it is evident that bicarbonate is essential in ACSF, but some ingenuity is necessary to prevent the loss of bicarbonate and to stabilize the $\mathrm{pH}$ of the solution. This may also be one of the reasons for the delay in the development of a commercially available ACSF. Before ARTCEREB was developed, we (Keio University Hospital) had prepared ACSF with almost the same composition and properties of ARTCEREB by mixing $20 \mathrm{~mL}$ (1 ampoule) of Meylon Injection 7\% [sodium bicarbonate concentration, $1.4 \mathrm{~g} / 20 \mathrm{~mL}$ (7\%); pH=ca. 7.9; osmotic ratio=ca. 5] with $500 \mathrm{ml}$ (1 bottle) of ACSF trial product No. 19 just prior to use. To stabilize the bicarbonate, ARTCEREB is packaged in an inner bag which is a double compartment bag, and a $\mathrm{CO}_{2}$ barrier free outer bag which prevents the escape of $\mathrm{CO}_{2}$. The inner bag, which is removed from the outer bag just prior to use, is a double compartment bag in which two kinds of solutions are separated by a septum. Both solutions are mixed by opening the septum with manual compression and the mixed solution (ARTCEREB) is administered by a direct drip to the operative field (closed system). ARTCEREB also retained its $\mathrm{pH}$, within the prescribed limit, for 6 hours in an open system (Figure 2). Thus, ARTCEREB can be safely used for longer operations by adding, or by replacing with, fresh solution. ARTCEREB has various improvements for the long-term stabilization of the $\mathrm{pH}$ of bicarbonatecontaining ACSF, including the adoption of a double compartment bag. No other ACSFs, including Elliott's solution and inhospital ACSF preparations, have had such great care taken to stabilize the $\mathrm{pH}$ [28]. Normal saline has been used instead of an appropriate irrigation and perfusion fluid in Japan (normal saline and Ringer's solution have only been approved for intravascular injection in Japan). Recently, perfusion fluids approved for the fields of ophthalmology, orthopedics, or urology have also been used as perfusion fluids for surgery.

\section{Basic experiments with rats}

We demonstrated that the mitochondrial activity of cultured brain cells exposed to ARTCEREB, measured by the rhodamine 123 method, was similar to intact cultured brain cells. However the mitochondrial activity of the lactated Ringer's solution and normal saline groups were reduced to $45 \%$ and $32 \%$, respectively, compared to intact cultured brain cells, indicating that these solutions have unfavorable or damaging effects on these cells [8]. Although the brain tissues around the operative field are injured by surgery, to a greater or lesser extent, the degree of damage in the surrounding tissues, such as edema, increased vascular permeability, and decreased mitochondrial activity, was less in the ARTCEREB group in comparison with the lactated Ringer's solution and normal saline groups [7]. In other words, ARTCEREB causes milder damage to brain cells compared with the other two solutions. Recently, the concept of minimally invasive surgery has been proposed, not just for neurological surgery but for all surgical fields. Any surgery, at least neurosurgery, which does not use ARTCEREB or ACSF as an irrigation and perfusion fluid cannot be called minimally invasive surgery.

\section{Clinical trials}

The purpose of any clinical trial is the ascertainment of safety, which is the most important issue for the clinical use of a new drug. Since the composition and properties of ARTCEREB are very similar to those of human CSF, the occurrence of adverse effects associated with its use is unimaginable. Even if signs or findings of adverse effects appear, it is impossible to attribute their cause to any of the components or properties of ARTCEREB.

However, since ARTCEREB contains bicarbonate, it will release $\mathrm{CO}_{2}$ and be alkalized when it comes into contact with the air. Thus, it is important to stabilize and maintain the $\mathrm{pH}$ during clinical trials and practical clinical use. Therefore, the inner bag of ARTCEREB is packed within a $\mathrm{CO}_{2}$ barrier free outer bag. Clinical trials of ARTCEREB were conducted with a closed system, and its efficacy (performance) and safety (adverse effects) were judged to be good or excellent in all patients.

There are no grounds for using normal saline or similar perfusion fluids that have harmful effects on neuronal cells for intraventricular perfusion in neuroendoscopic surgery. In addition, even if irrigation and perfusion fluids which have harmful effects on neuronal cells induce no immediate symptoms, the resultant neuronal damage may cause remote symptoms and impairment. It should be emphasized that neurosurgeons who intend to perform minimally invasive surgery should use ARTCEREB, or at least another ACSF, for neurological surgery.

\section{Conclusions}

Our basic experiments and clinical trials demonstrated the chemical 
Citation: Shiobara R, Ohira T, Doi K, Nishimura M, Kawase T (2013) Development of Artificial Cerebrospinal Fluid: Basic Experiments, and Phase II and III Clinical Trials. J Neurol Neurophysiol 4: 173. doi:10.4172/2155-9562.1000173

stability and safety of ARTCEREB, a commercially available ACSF, in the central nervous system. In particular, the clinical trials proved the excellent efficacy (performance) of ARTCEREB as an irrigation and perfusion fluid in neurological surgery. To improve surgical outcomes in the field of neurological surgery, especially when minimally invasive surgery is intended, ARTCEREB ought to be extensively used.

\section{Acknowledgments}

We thank the following 17 cooperated institutes and 157 patients in the clinical trial phase II and III: Ashikaga Red Cross Hospital, Fujita Health University Hospital, lizuka Hospital, Iwate Medical University Hospital, Jikei University Hospital, Juntendo University Hospital, Keio University Hospital, Kobe City Medical Center General Hospital, Kyushu University Hospital, Nakamura Memorial Hospital, National Cardiovascular Center, National Defense Medical College Hospital, Niigata University Medical \& Dental Hospital, Okayama University Hospital, Osaka University Hospital, Tokyo Medical University Hospital, Tokyo Women's Medical University Hospital.

This work was supported in part by Otsuka Pharmaceutical Factory, Inc, Tokushima, Japan.

\section{References}

1. ELLIOTT KA, JASPER HH (1949) Physiological salt solutions for brain surgery; studies of local $\mathrm{pH}$ and pial vessel reactions to buffered and unbuffered isotonic solutions. J Neurosurg 6: 140-152.

2. Shiobara R, Toya S, lisaka Y, Shizawa H, Ichikizaki K (1976) Early surgery of ruptured cerebral aneurysm in the acute stage (Jpn). Annual bulletin of Mitsukoshi health and welfare foundation. Tokyo 12: 37-49.

3. Shiobara R, Toya S, lisaka Y, Shizawa H, Ichikizaki K (1977) [An evaluation of the continuous ventricular drainage for ruptured cerebral aneurysms (author's transl)]. Neurol Med Chir (Tokyo) 17: 145-152.

4. Shiobara R, Toya S, lisaka Y, Ootani M, Kawase T (1983) Development and clinical use of artificial cerebrospinal fluid and ventriculo-cisternal irrigation fluid (Jpn). Jpn J Stroke. Proc 8th annual Japanese conference of stroke Tokyo 5: 196.

5. Shiobara R, Kawase T, Toya S, Ebato S, Miyahara Y (1986) Ventricular cisternal perfusion using artificial CSF with urokinase for subarachnoid hemorrhage (Jpn). Neurol med chirurg. Proc 45th annual meeting abstracts the Japan neurological society Tokyo 363

6. Shiobara R, Kawase T, Toya S, Ebato K, Miyahara Y (1985) Scavengery surgery for subarachnoid hemorrhage. II) Continuous ventriculo-cisternal perfusion by use of artificial cerebrospinal fluid with urokinase. In: Auer LM (ed.) Timing of Aneurysm Surgery. Walter de Gruyter Press: Berlin, New York, USA 365-372.

7. Doi K, Kawano T, Morioka Y, Fujita Y, Nishimura M (2006) Various irrigation fluids affect postoperative brain edema and cellular damage during experimental neurosurgery in rats. Surg Neurol 66: 565-572.

8. Nishimura N, Doi K, Enomoto R, Lee E, Naito S, et al. (2008) ARTCEREB Irrigation and perfusion solution for cerebrospinal surgery (Jpn). Pharmacological assessment using astrocytes exposed to test solution. Cell Industrial Info Tokyo 40: 254-257.

9. Shiobara R, Ohira T, Onozuka S, Kawase T (2005) A study of the use of irrigation fluid in neurosurgery (Jpn). Jpn J Neurosurg. Tokyo 14: 323-330.

10. Shiobara R, Ohira T, Onozuka S, Kawase T (2006) A study of the use of irrigation fluid in neurosurgery II (Jpn). J New Rem \& Clin Tokyo 55: 1040-1133.

11. Shiobara R, Kageji T, Nakagawara J, Murakami H, Ohira T (2007) Safety evaluation of ACF-95 for use as irrigation or perfusion solutions in neurosurgery patients: Phase II clinical trial (Jpn). J New Rem \& Clin Tokyo 56: 1404-1456.

12. Shiobara R, Kageji T, Kawase T, Nakagawara J, Arai K, et al. (2007) Multicenter study of ACF-95 for use as irrigation or perfusion solutions in patients who underwent burr hole opening, craniotomy surgery, or neuroendoscopic surgery: Phase III clinical trial (confirmatory study) (Jpn). J New Rem \& Clin. Tokyo 56: 1458-1523.

13. Davson $H(1967)$ Chemical composition and secretory nature of the fluid. In: Davson H Physiology of the cerebrospinal fluid. London: J \& A Churchill 33-54.

14. Milhorat $\mathrm{TH}(1972)$ Cerebrospinal fluid physiology. In: Hydrocephalus and the cerebrospinal fluid. Baltimore USA: Williams \& Wilkins 1-41.
15. Johnson LV, Walsh ML, Chen LB (1980) Localization of mitochondria in living cells with rhodamine 123. Proc Natl Acad Sci U S A 77: 990-994.

16. Marmarou A, Poll W, Shulman K, Bhagavan H (1978) A simple gravimetric technique for measurement of cerebral edema. J Neurosurg 49: 530-537.

17. Uyama O, Okamura N, Yanase M, Narita M, Kawabata K, et al. (1988) Quantitative evaluation of vascular permeability in the gerbil brain after transient ischemia using Evans blue fluorescence. J Cereb Blood Flow Metab 8: 282-284.

18. Preston E, Webster J (2000) Spectrophotometric measurement of experimental brain injury. J Neurosci Methods 94: 187-192.

19. World Medical Association: Helsinki declaration.

20. The Japanese Government (1960) Article 14, Paragraph 3 and Article 80, Paragraph 2 of Pharmaceutical Affairs Law.

21. Ministry of Health and Welfare, Japan (1997) Ordinance of Ministry of Health and Welfare for implementation standard for clinical tests of drugs.

22. Oka K, Yamamoto M, Nonaka T, Tomonaga M (1996) The significance of artificial cerebrospinal fluid as perfusate and endoneurosurgery. Neurosurgery 38: 733-736.

23. Kodama N, Sasaki T, Kawakami M, Sato M, Asari J (2000) Cisternal irrigation therapy with urokinase and ascorbic acid for prevention of vasospasm after aneurysmal subarachnoid hemorrhage. Outcome in 217 patients. Surg Neurol 53: $110-117$.

24. Ohira T, Toya S (1996) Rigid-rod neuroendoscope (Jpn). In: Sato O, Ohi S (eds.) Neuroendoscopic Surgery. Basic knowledge and surgical technique. Tokyo Miwa Shoten 60-64.

25. Ohira T, Toya S (1996) Navigation endo-microscope (Jpn). In: Sato O, Oh $\mathrm{S}$ (eds.) Neuroendoscopic Surgery. Basic knowledge and surgical technique. Tokyo: Miwa Shoten 101-104.

26. Ohira T, Kawase T (2001) Complication and countermeasure (Jpn). In Takakura K, Saito I, Kawase T, Teramoto A (eds.) Neurological Surgery. Advanced Practice. Neuroendoscopic Surgery Tokyo Medical View 122-124.

27. Enomoto R, Tatsuoka H, Komai T, Sugahara C, Takemura K, et al. (2004) Involvement of histone phosphorylation in apoptosis of human astrocytes after exposure to saline solution. Neurochem Int 44: 459-467.

28. Japanese Society of Hospital Pharmacists (2003) Artificial cerebrospinal fluid (Jpn), in Japanese Society of Hospital: In-hospital drug preparation. (5thedn), Tokyo Yakuji Nipposha 225-226.

29. Kamikawa H, Kobayashi N (1996) Shunt tube manipulation (Jpn), In: Sato $\mathrm{O}$, Ohi $\mathrm{S}$ (eds.) Neuroendoscopic Surgery. Basic knowledge and surgical technique. Tokyo Miwa Shoten 221-225.

30. Oka K, Yamamoto M, Nonaka T, Tomonaga M (2001) Hydrocephalus (Jpn). In Takakura K, Saito I, Kawase T, Teramoto A (eds.) Neuroendoscopic Surgery: Neurological Surgery. Advanced Practice 3. Tokyo Medical View 60-69.

31. ELLIOTT KA, LEWIS RC (1950) Clinical uses of an artificial cerebrospinal fluid J Neurosurg 7: 256-260.

32. Oka K, Tomonaga M (1996) Steerable/flexible neuroendoscope (Jpn). Neuroendoscopic Surgery: Basic knowledge and surgical technique. In: Sato O, Ooi S (eds.) Tokyo Miwa Shoten 69-72.

33. Oota T (2000) New classification of level of consciousness (Jpn). In: Oota T Matsutani M (eds.) Neurosurgery, (8thedn) Kyoto Jpn Kinpoudou 177-183.

This article was originally published in a special issue, Brain Tumor handled by Editor(s). Dr. Colleen Dockstader, The Hospital for Sick Children, Canada 